\title{
KIAA0101 knockdown inhibits cell proliferation and induces cell cycle arrest and cell apoptosis in chronic lymphocytic leukemia cells
}

\author{
Qing Zhang, Jingjing Yuan, Yanyan Liu, Xingchen Liu, Tianxin Lv, Keshu Zhou, Yongping Song \\ Department of Hematology, Affiliated Cancer Hospital of Zhengzhou University, Henan Cancer Hospital, Zhengzhou, China \\ Contributions: (I) Conception and design: Q Zhang, K Zhou, Y Song; (II) Administrative support: Y Song; (III) Provision of study materials or \\ patients: J Yuan, Y Liu; (IV) Collection and assembly of data: Q Zhang, T Lv; (V) Data analysis and interpretation: Q Zhang; (VI) Manuscript \\ writing: All authors; (VII) Final approval of manuscript: All authors. \\ Correspondence to: Yongping Song, MD, PhD. Department of Hematology, Affiliated Cancer Hospital of Zhengzhou University, Henan Cancer \\ Hospital, No. 127, Dongming Road, Jinshui District, Zhengzhou 450003, China. Email: songyongping001@126.com; Keshu Zhou, MD, PhD. \\ Department of Hematology, Affiliated Cancer Hospital of Zhengzhou University, Henan Cancer Hospital, No. 127, Dongming Road, Jinshui \\ District, Zhengzhou 450003, China. Email: drzhouks77@163.com.
}

\begin{abstract}
Background: Chronic lymphocytic leukemia (CLL) is a heterogeneous disease with intense cytogenetic aberrations. Importantly, our recent report indicated that thyroid hormone receptor interactor 13 (TRIP13) is a potential new therapeutic target in CLL. In this study, we predicted 20 TRIP13-related genes and found that KIAA0101 is a novel gene that regulates cell proliferation and the cell cycle of CLL cells.

Methods: CD19+ $\mathrm{B}$ cells were isolated from the peripheral blood of 26 CLL patients and 6 healthy donors through magnetic cell sorting. Cell proliferation was assessed by the CCK- 8 assay. The mRNA and protein levels of genes were examined through RT-qPCR and western blot assays, respectively. Cell cycle and cell apoptosis were measured through Annexin V-based flow cytometry and the caspase 3/7 activity assay. Potential targets of KIAA0101 were identified through microarray analysis. 20 TRIP13 related genes was predicted by Ingenuity Pathway Analysis (IPA). KIAA0101-regulated functions and molecular pathways were predicted through IPA.

Results: KIAA0101 knockdown had the strongest inhibitory effect on CLL cell proliferation among the 20 TRIP13-related genes. KIAA0101 was highly expressed in CD19+ B cells of CLL patients. KIAA0101 knockdown induced cell cycle arrest and cell apoptosis, and inhibited FOXO1, MYD88, and TLR4 expression in CLL cells.

Conclusions: Taken together, we demonstrated that KIAA0101 plays a critical role in cell proliferation and the cell cycle of human CLL cells. KIAA0101 knockdown induced cell apoptosis, and reduced FOXO1, MYD88, and TLR4 expression, and may therefore be used as a therapeutic target of CLL.
\end{abstract}

Keywords: Chronic lymphocytic leukemia (CLL); KIAA0101; proliferation; apoptosis; cycle

Submitted Jan 18, 2021. Accepted for publication Mar 20, 2021.

doi: $10.21037 / \mathrm{atm}-21-626$

View this article at: http://dx.doi.org/10.21037/atm-21-626

\section{Introduction}

Chronic lymphocytic leukemia (CLL), a type of B-cell malignancy that is incurable, is the most common type of leukemia in the Western hemisphere (1). Over the past decades, significant advances have been made in the treatment of CLL following the introduction of small molecule inhibitors (2-5). For instance, ibrutinib, which is currently approved for the front-line treatment of CLL, induces long-lasting responses in the majority of patients, improving outcomes with relatively limited toxicity (6). 
However, small molecule inhibitors still have potential problems such as drug resistance, which restrict the efficacy of CLL treatment (7). Hence, it is imperative to explore the cellular and molecular mechanisms of CLL tumorigenesis and progression.

Thyroid hormone receptor interactor 13 (TRIP13) has been found to be implicated in mitotic and DNA repair processes and the pathogenesis of multiple cancers (8-11). For instance, TRIP13 knockdown inhibited cell proliferation and invasion, and induced cell apoptosis and cell cycle arrest in vitro, while TRIP13 overexpression promoted xenograft tumor growth in vivo in head and neck cancer (8). High genomic copy number of TRIP13 was observed in the majority of non-small cell lung cancers (9). In addition, our previous study demonstrated that TRIP13 knockdown inhibited CLL cell proliferation and induced cell apoptosis (10). Considering the vital roles of TRIP13 in tumor malignant transformation, including CLL (8-11), we speculated that TRIP13-related genes might also play vital roles in CLL tumorigenesis and progression.

In this study, 20 TRIP13 downstream genes (GOLGA7B, HIST1H4B, OTUB2, SEZ6L2, SMC2, EPSTI1, HIST1H4C, OLFML2A, SHISA2, DEPDC1, FBXO43, HPS 3, KIAA0101, KNL1, MAP9, PIF1, RFWDs3, SAPCD2, $S N X 10$, and UBE2T) were identified through the Ingenuity Pathway Analysis (IPA) database (10). Next, lentiviruses that could interfere with the expression of these genes were constructed, and the effects of knockdown of these genes on B-cell lymphocytic leukemia cell (Granta-519) proliferation were examined. Among these genes, KIAA0101 mRNA abundance was relatively high in Granta-519 cells, and KIAA0101 knockdown had the strongest inhibitory effect on Granta-519 cell proliferation. KIAA0101, also known as proliferating cell nuclear antigen (PCNA)associated factor (PAF15), is a conserved PCNA-binding protein (12). KIAA0101 involved in DNA replication and damage repair process by mediating the interaction with DNA synthesis polymerase (12). Prior studies showed that KIAA0101 might play vital roles in the development and progression of various cancers. For instance, KIAA0101 has been found to be involved in microvascular invasion and epithelial-mesenchymal transition of hepatocellular cancer (13), anchorage-independent growth and migration of fibrosarcoma (14), cell cycle regulation of gastric cancer (15), and cell migration and chemoresistance of ovarian cancer (16). Nevertheless, the biological functions of KIAA0101 along with related molecular mechanisms of action in CLL remain to be elucidated.

In this study, downstream genes of KIAA0101 were identified through microarray analysis. Pathway, function, and disease enrichment analyses for KIAA0101-regulated genes were performed using the IPA database. Effects of KIAA0101 knockdown on cell proliferation, cell cycle progression, and cell apoptosis were examined in CLL cell models (Granta-519 and JVM-2 cell lines). Also, the effects of KIAA0101 loss on the expression of genes of interest (FOXO1, MYD88, TLR4) were further assessed through western blot assays in Granta-519 cells.

We present the following article in accordance with the MDAR reporting checklist (available at http://dx.doi. org/10.21037/atm-21-626).

\section{Methods}

\section{Clinical samples}

Blood samples of 26 CLL patients and 6 healthy people were collected at the Affiliated Cancer Hospital of Zhengzhou University (Henan Cancer Hospital, Henan, China) during March 2017 to December 2018. CD19+ B cells were freshly isolated from peripheral blood mononuclear cells (PBMCs) through magnetic cell sorting (MACS) of Lymphoprep-separated buffy coats based on MACS CD19 Microbeads (Miltenyi Biotec, Bergisch Gladbach, Germany) (>98\%). All procedures performed in this study involving human participants were in accordance with the Declaration of Helsinki (as revised in 2013). The study was approved by the Ethics Committee of the Henan Cancer Hospital (No. 2016098). Written informed consent was obtained from all participants.

\section{Cell lines}

JVM-2 is an Human Epstein-Barr virus (EBV)-transformed B-lymphocytic leukemia (B-CLL) cell line (17). Granta-519 is a Human mantle cell lymphoma cell line, but functionally similar to CLL cells (18). JVM-2 and Granta-519 cell lines were supplied by the cell bank of Institute of Hematology, Chinese Academy of Medical Sciences (CAMS) (Tianjin, China) and were cultured in DMEM medium (Thermo Fisher Scientific, Rockford, IL, USA) supplemented with $10 \%$ fetal bovine serum (FBS, Gibco, Thermo Fisher Scientific) at $37^{\circ} \mathrm{C}$ in a $95 \%$ air $/ 5 \% \mathrm{CO}_{2}$ atmosphere. 
Table 1 Sequences of primers (5' to $3^{\prime}$ ) and shRNA used in this study

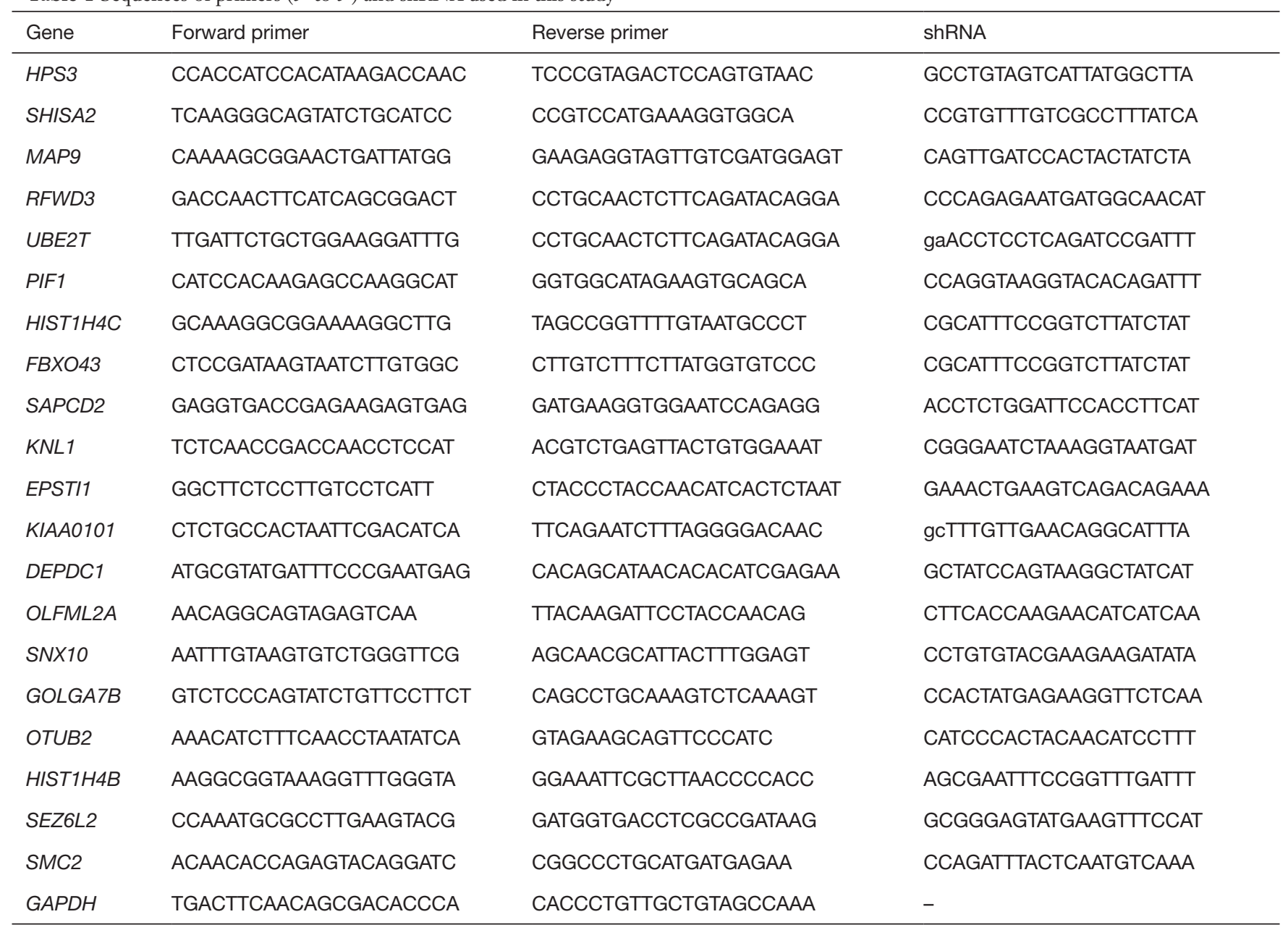

\section{Real-time quantitative PCR (RT-qPCR)}

Total RNA was extracted from cells using Trizol reagent (Thermo Fisher Scientific, MA, USA) following the manufacturer's instructions. Synthesis of cDNA was carried out through a reverse transcription reaction using M-MLV Reverse Transcriptase (Promega, Madison, WI, USA) on an Applied Biosystems 2720 Thermal Cycler (Thermo Fisher Scientific). Subsequently, cDNA was amplified and quantified using the SYBR Green kit (Toyobo, Osaka, Japan) and specific quantitative primers on the TAKARA TP800-Thermal Cycler Dice ${ }^{\mathrm{TM}}$ Real Time System (Takara, Osaka, Japan). Real-time quantitative PCR reactions were performed with the following cycling parameters: $95^{\circ} \mathrm{C}$ for $30 \mathrm{~s}, 45$ cycles of $95^{\circ} \mathrm{C}$ for $5 \mathrm{~s}$, and $60{ }^{\circ} \mathrm{C}$ for $30 \mathrm{~s}$. All sequences of the primers are listed in Table 1 .

\section{Western blot assay}

Whole-cell lysates were prepared from CLL cells using RIPA buffer (Sigma-Aldrich, St. Louis, MO, USA). After quantification, an equal amount of proteins were separated by sodium dodecyl sulfate-polyacrylamide gel electrophoresis (SDS-PAGE). Proteins in the gel were transferred to nitrocellulose membranes. Next, the membranes were incubated overnight at $4{ }^{\circ} \mathrm{C}$ with primary antibodies against $F O X O 1, p 53$, and $C D K 6$ (\#2880, \#2524, and \#3136, respectively, Cell Signaling Technology, Danvers, MA, USA) (19); Bax, TCF4, MYD88, and TLR4 (ab7977, ab185736, ab2064, and ab13556, respectively, Abcam, Cambridge, UK), and glyceraldehyde 3-phosphate dehydrogenase (GAPDH, sc-32233, Santa Cruz Biotechnology, Dallas, Texas, USA). The membranes 
were then probed with a horseradish peroxidase-labeled secondary antibody (sc-2004/sc-2005, Santa Cruz Biotechnology) for $1 \mathrm{~h}$ at room temperature. Finally, protein signals were detected through chemiluminescence using ECL Western Blotting Detection Reagents (GE Healthcare, Pittsburgh, PA, USA) and visualized through the G:BOX Chemi Gel Documentation System (Syngene, Frederick, MD, USA).

\section{Plasmids and lentiviral transduction}

Recombinant lentiviruses were produced by Shanghai Genechem Company (Shanghai, China). The shRNA sequence (5'-CCGGGC-target sequence-CTCGAGreverse complementary target sequence-GCTTTTTG-3') was subcloned into the lentiviral silencing vector. A scramble shRNA (5'-GCCTAACTGTGTCAGAAGGAA-3') served as the negative control (ctrl). When cells were grown to approximately $40 \%$ confluence, cells were infected with lentiviruses at the multiplicity of infection (MOI) of 50-100. All sequences of the shRNA are listed in Table 1.

\section{Cell Counting Kit-8 assay}

Infected cells were seeded into $96-$-well plates $(2,000$ cells per well). At the indicated time points after infection, $10 \mu \mathrm{L}$ of Cell Counting Kit-8 (CCK-8) solutions (Sigma-Aldrich) was added into each well. After $4 \mathrm{~h}$ of incubation at $37^{\circ} \mathrm{C}$, cell absorbance was measured at $450 \mathrm{~nm}$.

\section{Apoptosis assay}

Cell apoptotic rate was measured after Annexin V-APC staining using flow cytometry. Briefly, cells were collected on day 5 after lentivirus infection and re-suspended in binding buffer at a concentration of $1 \times 10^{6}$ cells $/ \mathrm{ml}$. Next, $100 \mu \mathrm{L}$ of cell suspensions were co-incubated with Annexin $\mathrm{V}$-APC staining solution for $15 \mathrm{~min}$ in the dark at room temperature. Finally, cell apoptotic patterns were analyzed using flow cytometry.

\section{Caspase 3/7 activity detection}

Caspase 3/7 activity in CLL cells was detected using the Promega Caspase-Glo 3/7 assay kit (Promega). Briefly, cells infected with shctrl or shKIAA0101 lentiviruses were plated into 96-well plates and incubated for 5 days. Next, cells were collected and cell suspensions $\left(1 \times 10^{4}\right.$ cells/well $)$ were reacted with $100 \mu \mathrm{L}$ of Caspase-Glo reaction solution. After shaking for $30 \mathrm{~min}$ at 300-500 rpm, the reaction system was incubated for $2 \mathrm{~h}$ at room temperature. Finally, signal intensity was determined using a microplate reader.

\section{Gene microarray analysis}

Total RNA was extracted from Granta-519 cells infected with shctrl or shKIAA0101 lentiviruses. Next, the content and quality of RNA were analyzed using the Nanodrop 2000 (Thermo Fisher Scientific) and the Agilent 2100 Bioanalyzer (Agilent, Pal Alto, CA, USA). RNA (1.7< A260/ A280 $<2.2$, RNA integrity number $\geq 7.0$ and $28 \mathrm{~S} / 18 \mathrm{~S}>0.7)$ was reverse transcribed into first-strand cDNA, which was further converted into double-stranded DNA and biotinlabeled amplified RNA (aRNA) using the GeneChip 3' IVT Express Kit (Affymetrix, Santa Clara, CA, USA). After purification, aRNA was quantified using the Nanodrop 2000 (Thermo Fisher Scientific), fragmented, and hybridized with the GeneChip Human Genome U133 plus 2.0 Array (Affymetrix). Next, hybridized chips were stained and washed using the GeneChip Hybridization Wash and Stain Kit (Affymetrix) on the Genechip Fluidics Station 450 instrument (Affymetrix). Finally, microarray signals were scanned and analyzed using the Genechip Array Scanner $30007 \mathrm{G}$ (Affymetrix).

\section{Bioinformatics analysis}

The IPA database was used to examine the potential functions and regulatory mechanisms of differentially expressed genes in the shKIAA0101 group vs. the shctrl group through "disease and function", "canonical pathway", and "interaction network" analysis.

\section{Statistical analysis}

Data analysis was performed using GraphPad Prism 7 software (GraphPad Software, La Jolla, CA, USA). The results are shown as mean \pm standard deviation $(\mathrm{SD})(\mathrm{n}=3)$. Statistical significance was determined by the $t$ test between data from two groups, and Two-way ANOVA was used to evaluate the differences among multiple groups. Results were regarded as statistically significant when the $\mathrm{P}$ value 

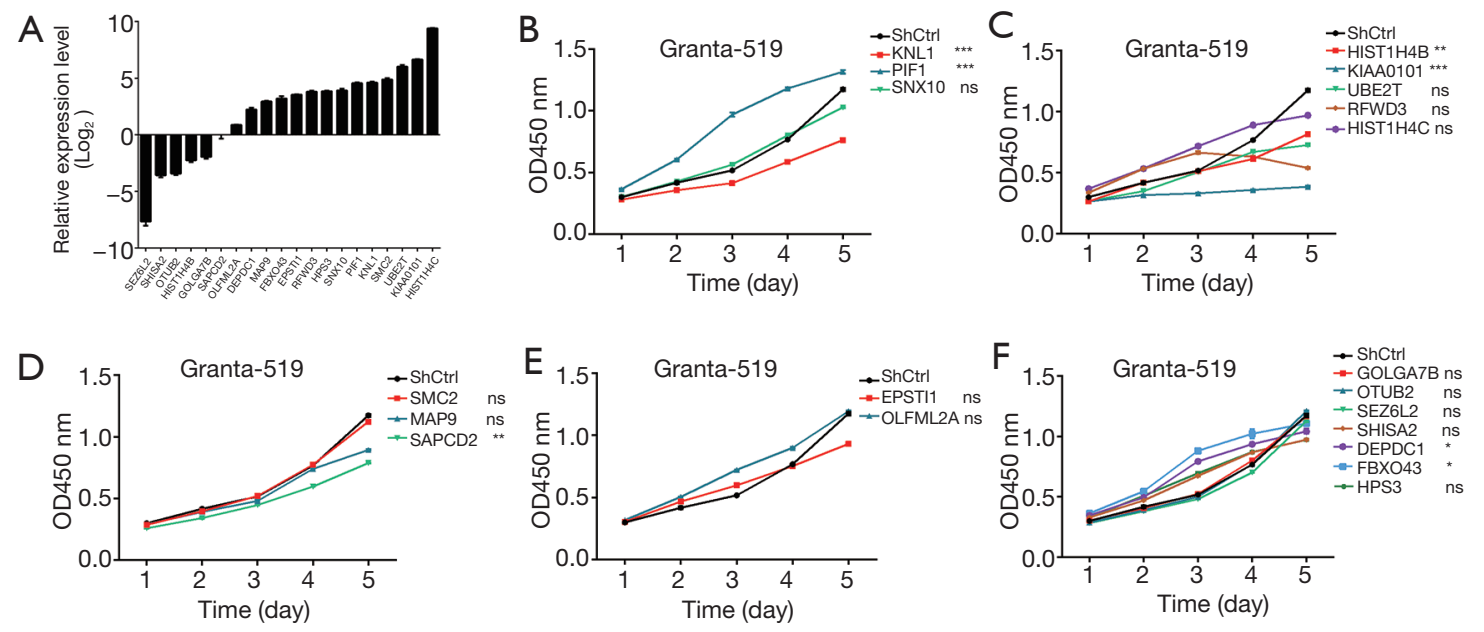

Figure 1 Effects of knockdown of 20 TRIP13-related genes on Granta-519 cell proliferation. (A) The mRNA abundances of 20 TRIP13related genes predicted by the IPA database in Granta-519 cells were examined through RT-qPCR. The expression levels of all genes were normalized to SAPCD2. (B,C,D,E,F) Granta-519 cells were infected with lentiviruses targeting the above-mentioned 20 genes or control lentiviruses (shctrl). The above-mentioned 20 genes were divided into 5 groups (i.e., chromosome maintenance (B), DNA repair (C), cell division (D), macrophage polarization (E), and others (F) according to their molecular functions. Cell proliferative ability was assessed through the CCK-8 assay at the indicated time points after infection. ${ }^{\text {ns }} \mathrm{P}>0.05,{ }^{*} \mathrm{P}<0.05,{ }^{* *} \mathrm{P}<0.01,{ }^{* * *} \mathrm{P}<0.001$. CLL, chronic lymphocytic leukemia; BTK, Bruton's tyrosine kinase; TRIP13, thyroid hormone receptor interactor 13; IPA, Ingenuity Pathway Analysis; RT-qPCR, real-time quantitative PCR; MACS, magnetic cell sorting; NC, negative control; CCK-8, Cell Counting Kit-8; aRNA, amplified RNA; MYD88, myeloid differentiation factor 88; TLR, toll-like receptor; FOXO, Forkhead box O.

was lower than 0.05 .

\section{Results}

\section{Effects of TRIP13-related genes on CLL cell proliferation}

Our previous study showed that TRIP13 knockdown inhibited cell proliferation and facilitated cell apoptosis in CLL (10). To further explore potential crucial genes implicated in the pathogenesis of CLL, 20 putative TRIP13-related genes were predicted through IPA, and the mRNA abundances of these genes in JVM-2 cells were measured using RT-qPCR. As presented in Figure 1A, KIAA0101 and HIST1H4C were relatively highly expressed among these genes in JVM-2 cells. Next, the effects of knockdown of these genes on JVM-2 cell proliferation were examined using the CCK- 8 assay. Results showed that knockdown of PIF1, KNL1, HIST1H4B, KIAA0101, $S A P C D 2, D E P D C 1$, and FBXO43 could markedly influence JVM-2 cell proliferation, while the loss of other genes did not have a significant effect on JVM-2 cell proliferation $\left({ }^{\mathrm{ns}} \mathrm{P}>0.05,{ }^{*} \mathrm{P}<0.05,{ }^{* *} \mathrm{P}<0.01\right.$, ${ }^{* *} \mathrm{P}<0.001$, Figure $1 B, C, D, E, F)$. Considering that KIAA0101 knockdown had the strongest inhibitory effect on JVM-2 cell proliferation, the functions of KIAA0101 in the development of CLL cells along with related molecular mechanisms were further investigated in subsequent experiments.

\section{KIAA0101 expression was markedly up-regulated in $C D 19^{+} B$ cells isolated from the peripheral blood of CLL patients}

Next, the mRNA expression pattern of KIAA0101 in CD19+ B cells isolated from the peripheral blood of 6 healthy donors and 26 CLL patients was examined through RTqPCR. Results showed that the mRNA level of KIAA0101 was markedly increased in the CLL group compared to the healthy control group $\left({ }^{*} \mathrm{P}<0.05\right.$, Figure 2$)$, suggesting the close link between KIAA0101 and CLL progression.

\section{Potential functions and molecular mechanism investigations of KIAA0101}

To further explore the functions and molecular mechanisms of KIAA0101 in CLL, Affymetrix GeneChip analysis was 


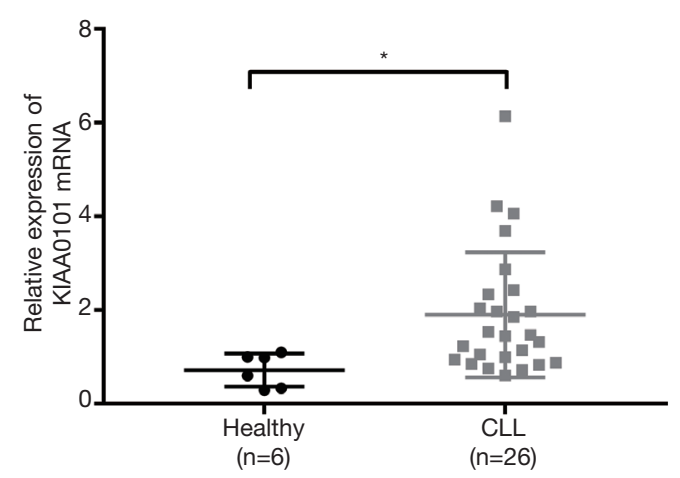

Figure 2 KIAA0101 expression was markedly up-regulated in $\mathrm{CD} 19^{+} \mathrm{B}$ cells isolated from the peripheral blood of CLL patients. The mRNA expression level of KIAA0101 was examined through RT-qPCR in CD19+ B cells isolated from the peripheral blood of 6 healthy people and $26 \mathrm{CLL}$ patients. ${ }^{*} \mathrm{P}<0.05$. CLL, chronic lymphocytic leukemia; RT-qPCR, real-time quantitative PCR.

performed to identify differentially expressed genes in Granta-519 cells infected with shKIAA0101 lentiviruses versus cells infected with shctrl lentiviruses. As presented in Figure 3A, 785 genes were differentially expressed (253 up-regulated, 532 down-regulated) in the shKIAA0101 group versus the shctrl group (I fold changel $>1.5$, $\mathrm{P}<0.05)$. Next, the IPA database was used to analyze the enrichment patterns of these differentially expressed genes in pathway, disease, and function categories. Disease and function enrichment analysis revealed that these differentially expressed genes were significantly enriched in cancers, and were mainly involved in the regulation of cell death, survival, and cell cycle (Figure $3 B$ ), suggesting that KIAA0101 might influence CLL tumorigenesis and progression principally through regulating the expression of genes related to cell cycle progression, cell survival, and cell death. IPA pathway enrichment analyses demonstrated that these differentially expressed genes were significantly enriched in multiple molecular pathways, especially in the p53/ATM signaling pathway and pathways associated with cancer, DNA damage/repair, cell cycle, and cell apoptosis (Figure 3C). These outcomes suggested that KIAA0101 might exert its functions through regulating the expression of genes implicated in the aforementioned pathways.

\section{Knockdown of KIAA0101 inbibited cell proliferation and induced cell cycle arrest in Granta-519 and $\mathcal{F V M - 2}$ cells}

Knockdown efficiency analysis revealed that the infection of shKIAA0101 lentiviruses triggered the marked downregulation of KIAA0101 mRNA and protein expression in Granta-519 and JVM-2 cells compared to the shctrl group $(\mathrm{P}<0.001$, Figure $4 A, B)$, suggesting that shKIAA0101 lentiviruses could be used for subsequent loss-of-function experiments. Functional analyses revealed that KIAA0101 knockdown strikingly inhibited cell proliferation in both Granta-519 and JVM-2 (Figure 4C,D) cells. Moreover, the percentages of cells in the G1 and G2/M phases were notably reduced and the proportion of cells in $\mathrm{S}$ phase was noticeably increased in Granta-519 and JVM-2 infected with shKIAA0101 lentiviruses relative to the shctrl group, suggesting that KIAA0101 knockdown induced cell cycle arrest at the $\mathrm{S}$ phase in Granta-519 and JVM-2 cells $\left({ }^{*} \mathrm{P}<0.05,{ }^{* *} \mathrm{P}<0.01,{ }^{* * *} \mathrm{P}<0.001\right.$, Figure $\left.4 E, F\right)$.

\section{KIAA0101 knockdown induced cell apoptosis in Granta-519 and $\mathcal{F V M - 2}$ cells}

Next, the effect of KIAA0101 knockdown on cell apoptosis was further investigated in Granta-519 and JVM-2 cells. Our outcomes revealed that KIAA0101 knockdown led to significant up-regulation of caspase $3 / 7$ activity in Granta-519 and JVM-2 cells compared with the shctrl group $(\mathrm{P}<0.001$, Figure $5 A, B)$. Also, the expression level of the anti-apoptotic protein Bcl-2 was strikingly reduced in Granta-519 and JVM-2 cells infected with shKIAA0101 lentiviruses compared to the shctrl group $(\mathrm{P}<0.001$, Figure 5C,D). Additionally, the percentage of apoptotic cells was notably elevated in the shKIAA0101 group $(12.12 \% \pm 0.3 \%$ in Granta- 519 and $7.47 \% \pm 0.81 \%$ in JVM- 2 ) compared to the shctrl group $(4.65 \% \pm 0.14 \%$ in Granta- 519 and $4.71 \% \pm 0.71 \%$ in JVM-2) (Figure $5 E, F$ ). In summary, these outcomes showed that KIAA0101 knockdown could induce cell apoptosis in Granta-519 and JVM-2 cells.

\section{KIAA0101 knockdown triggered the notable down- regulation of FOXO1, MYD88, and TLR4 protein levels in Granta-519 cells}

It is well known that the p53 signaling pathway is involved in the regulation of the cell cycle, cell apoptosis, and tumorigenesis. The interaction networks of KIAA0101 (PCLAF) and some KIAA0101 downstream genes of interest (BAX, FOXO1, CCND1, BRCA1, CDKN1A, CAST, CDK6, MYD88, TCF4, TLR4, and $p 53)$ were established using IPA interaction analysis (Figure 6A). Moreover, the effects of KIAA0101 knockdown on the expression of FOXO1, 
A

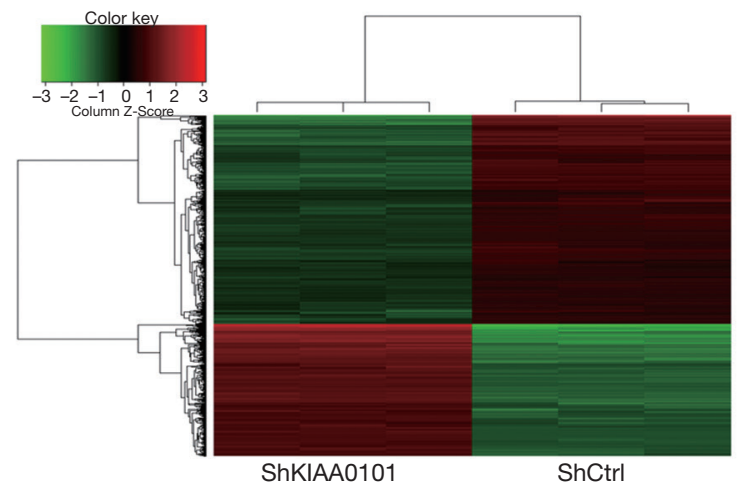

B
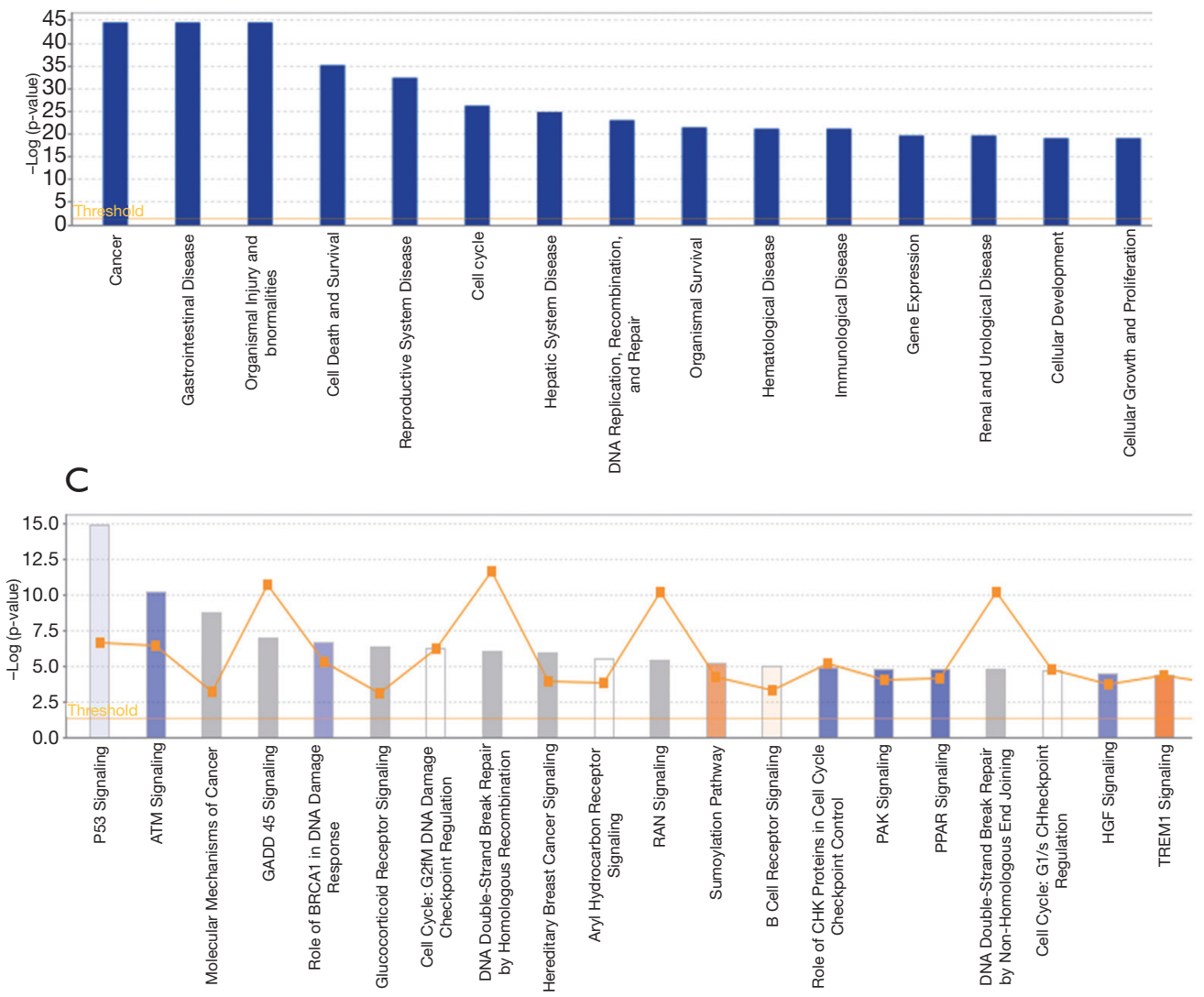

Figure 3 Potential functions and molecular mechanism investigations of KIAA0101. (A) Heatmap of differentially expressed genes (I fold changel $>1.5, \mathrm{P}<0.05$ ) in Granta-519 cells infected with shKIAA0101 lentiviruses versus cells infected with shctrl lentiviruses (green: downregulated; red: up-regulated). shKIAA0101 and shctrl groups contained 3 replicates. Genes and samples were displayed in rows and columns, respectively. (B) The top 15 disease and molecular function terms significantly enriched by differentially expressed genes. (C) The top 20 molecular functional pathways significantly enriched by differentially expressed genes.

MYD88, TCF4, and TLR4 were further examined through western blot assays. Consistent with our microarray data, western blot outcomes validated that KIAA0101 loss led to the notable down-regulation of FOXO1, MYD88, and TLR4 protein levels in Granta-519 cells (Figure 6B). These outcomes suggested that KIAA0101 might exert its functions partly through regulating FOXO1, MYD 88 , and TLR4 expression in Granta-519 cells. 

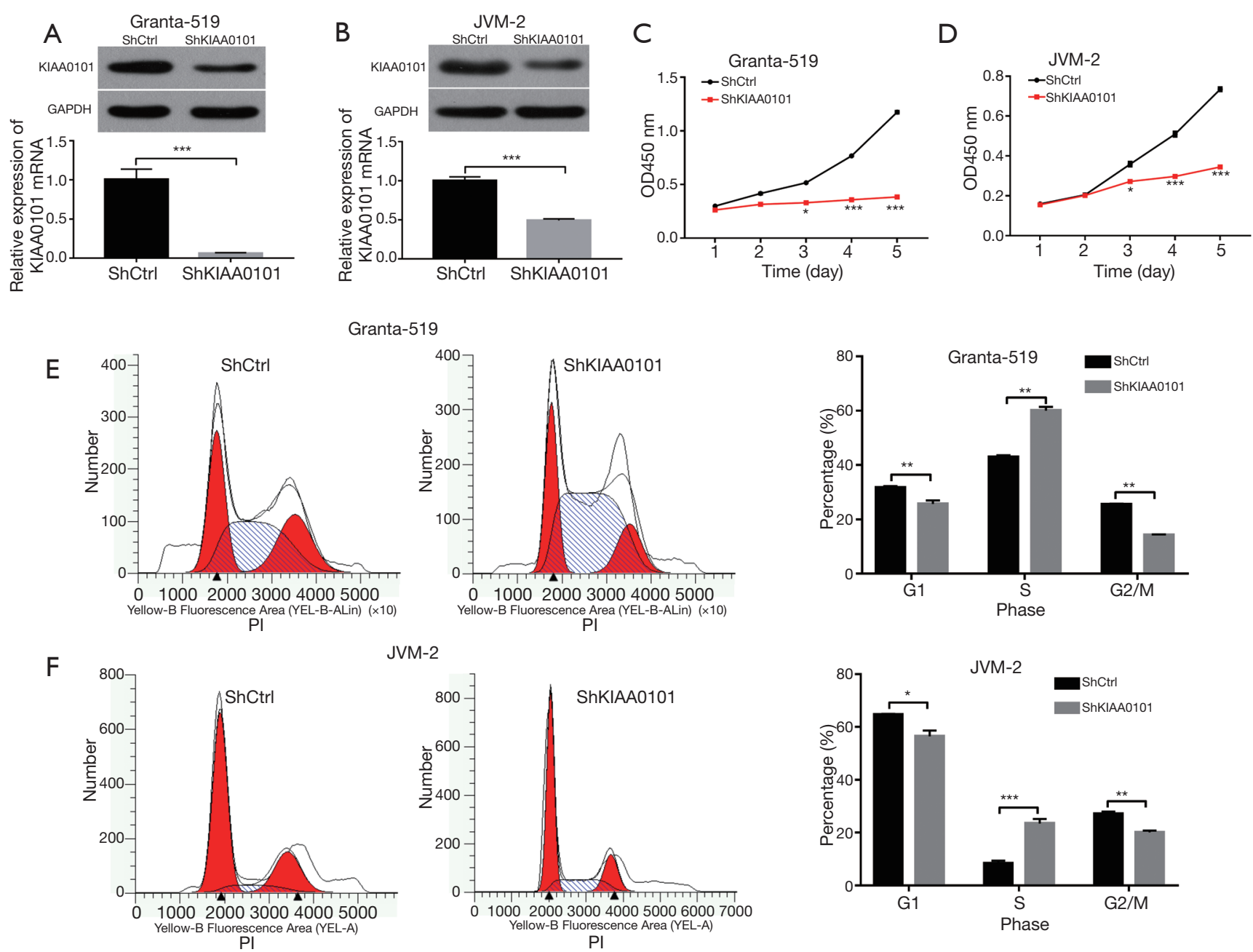

Figure 4 Knockdown of KIAA0101 inhibited cell proliferation and induced cell cycle arrest in Granta-519 and JVM-2 cells. (A,B,C,D,E,F) Granta-519 and JVM-2 cells were infected with shctrl or shKIAA0101 lentiviruses. (A,B) On day 3 after infection, mRNA and protein levels of KIAA0101 were measured through RT-qPCR and western blot assays, respectively. (C,D) At the indicated time points (1, 2, 3, 4, or 5 days) after infection, cell proliferative activity was detected by the CCK-8 assay. (E,F) On day 3 after infection, cell distribution patterns in different phases of the cell cycle were measured by flow cytometry after PI staining. ${ }^{*} \mathrm{P}<0.05,{ }^{* *} \mathrm{P}<0.01,{ }^{* *} \mathrm{P}<0.001$. RT-qPCR, real-time quantitative PCR; PI, propidium iodide.

\section{Discussion}

Considering the vital roles of TRIP13 in the development of CLL as shown in our previous study (10), we speculated that TRIP13-related genes might also be implicated in the pathogenesis of CLL. In this study, 20 TRIP13related genes were identified by IPA. Further investigations showed that KIAA0101 mRNA abundance was higher in Granta-519 cells, and KIAA0101 knockdown had the strongest inhibitory effect on Granta-519 cell proliferation. Moreover, a notable increase in KIAA0101 mRNA expression was observed in CD $19^{+} \mathrm{B}$ cells isolated from CLL patients compared to the healthy control group. These data suggest the close link between KIAA0101 and CLL progression. To gain a deeper insight into the functions and molecular mechanisms of KIAA0101 in CLL, gene microarray analysis was performed in Granta-519 cells infected with shKIAA0101 or shctrl lentiviruses. Through gene microarray analysis, we identified 785 potential downstream targets of KIAA0101. IPA database analyses further revealed that these possible targets of 

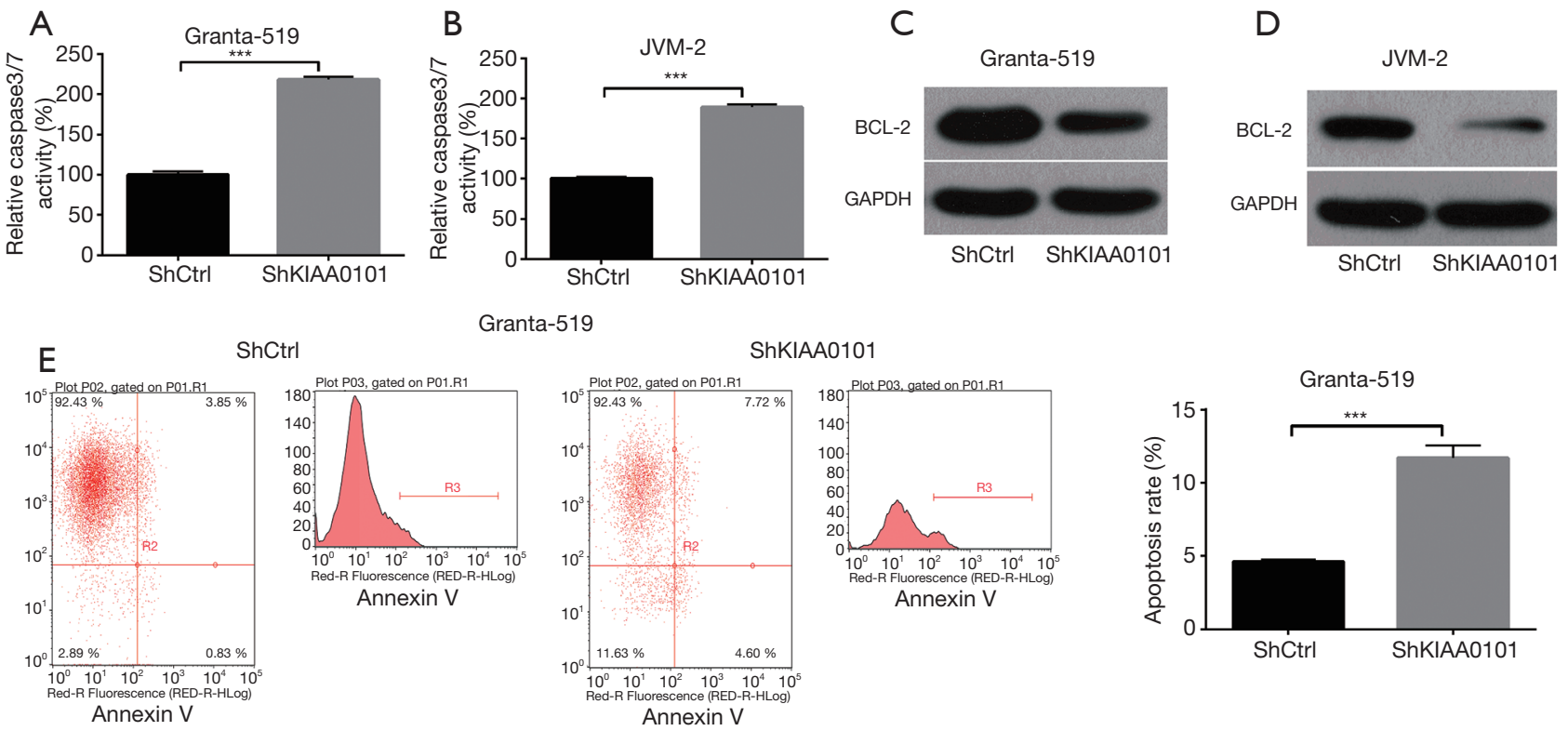

JVM-2

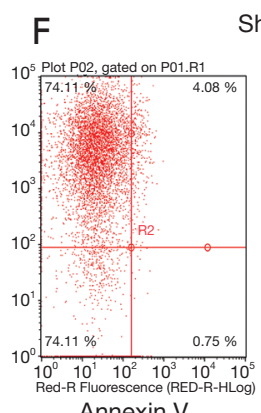

ShCtrl
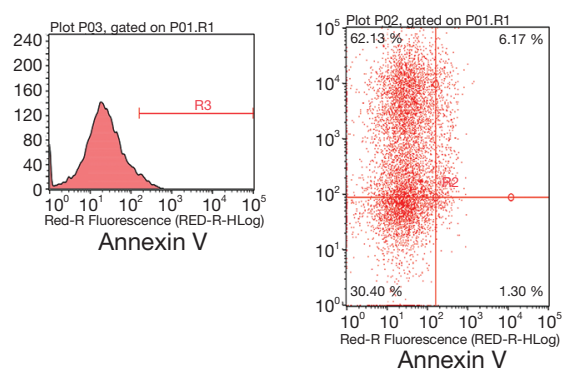

ShKIAA0101
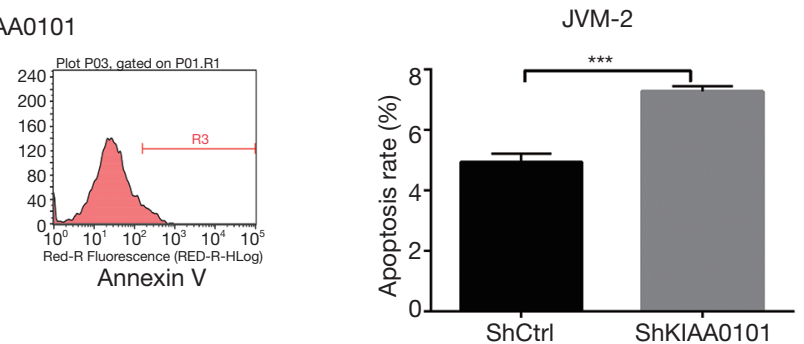

Figure 5 KIAA0101 knockdown induced cell apoptosis in Granta-519 and JVM-2 cells. (A,B,C,D,E,F) Granta-519 and JVM-2 cells were infected with shctrl or shKIAA0101 lentiviruses. (A,C) On day 3 post lentivirus infection, relative caspase 3/7 activity was determined by ELISA. (B,D) On day 3 post lentivirus infection, Bcl-2 protein level was detected by western blot assay. (E,F) On day 3 post lentivirus infection, cell apoptotic rate was assessed by flow cytometry. ${ }^{* * *} \mathrm{P}<0.001$.

KIAA0101 were mainly involved in the regulation of cancer progression, cell death/survival/cycle, and DNA damage/ repair. Hence, the effects of KIAA0101 knockdown on CLL cell proliferation, cell cycle progression, and cell apoptosis were investigated in subsequent experiments. Results showed that KIAA0101 loss weakened cell proliferative ability, hindered cell cycle progression, and induced cell apoptosis in Granta-519 and JVM-2 cells. Consistent with our outcomes, KIAA0101 has been found to be a potential oncogene in multiple cancers such as hepatocellular cancer (13), ovarian cancer (14), and breast cancer (20).

The toll-like receptor 4 (TLR4)/myeloid differentiation factor 88 (MYD88) signaling pathway has been found to be implicated in the tumorigenesis and progression of multiple cancers, including CLL $(21,22)$. For instance, MYD88 knockdown inhibited cell proliferation, migration, and invasion in vitro, and inhibited subcutaneous xenograft tumor growth in vivo in colorectal cancer (23). TLR4 and MYD88 were highly expressed in highly invasive human MDA-MB-231 breast cancer cells and breast cancer tissues, and TLR4 and MYD88 expression was positively associated with the histological grade and metastatic potential of breast cancer (24). In addition, previous studies showed that the inhibition of the MYD88 downstream signaling pathway induced CLL cell death and inhibited tumor development in CLL E $\mu$-TCL1 adoptive transfer models (22). In this 

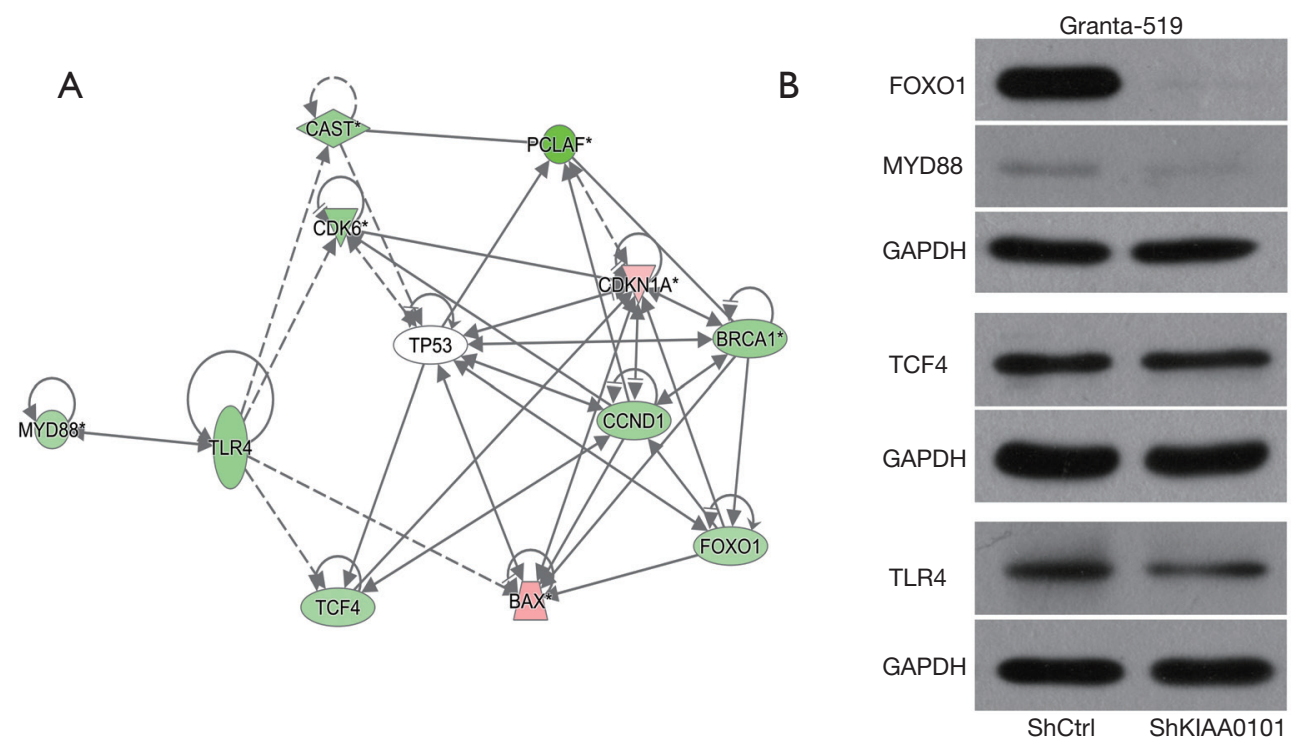

Figure 6 KIAA0101 knockdown triggered the notable down-regulation of FOXO1, MYD88, and TLR4 protein levels in Granta-519 cells. (A) The interaction networks of KIAA0101 (PCLAF) and some KIAA0101 downstream genes of interest (BAX, FOXO1, CCND1, BRCA1, CDKN1A, CAST, CDK6, MYD88, TCF4, TLR4, and P53) were established using the Ingenuity Pathway Analysis (IPA) database. (B) The effects of KIAA0101 knockdown on the expression of FOXO1, MYD88, TCF4, and TLR4 were examined through western blot assays on day 3 after lentivirus infection in Granta-519 cells infected with shctrl or shKIAA0101 lentiviruses.

study, our microarray and western blot data revealed that KIAA0101 knockdown led to the notable down-regulation of TLR4 and MYD88 protein levels in Granta-519 cells. These findings suggested the molecular mechanism of KIAA0101 -mediated regulation of cell proliferation may be realized through TLR4/MYD88 signaling pathway and this phenomenon is worthy of clarification in the future.

The Forkhead box O (FOXO) family have also been found to be crucial players in cell fate decisions and cancer development (25). In addition, previous studies showed that FOXO1 functioned as a tumor suppressor in multiple cancers such as breast cancer (26), non-small cell lung cancer (27), and gastric cancer (28). For example, ectopic expression of FOXO1 curbed cell proliferation, hindered cell cycle progression, and induced cell apoptosis in cervical cancer (29). Also, previous studies showed that FOXO1 expression was markedly up-regulated in freshly isolated CLL cells from peripheral blood and lymph node biopsies from CLL patients with poor prognosis (30). suggesting the close link between FOXO1 and CLL progression. Our present study also demonstrated that FOXO1 expression was markedly down-regulated in CLL cells following KIAA0101 knockdown. Interestingly, the functions of KIAA0101 were similar to an oncogene in CLL based on promoted cell proliferation, silencing of KIAA0101 leads to dramatic CLL cells apoptosis, cell cycle arrest and inhibition of cell proliferation. However, knock down of KIAA0101 also decreased the expression of FOXO1 which served as tumor suppressor genes as reported. According to these findings, FOXO1 expression might be independent on cell proliferation in KIAA0101-depleted CLL cells.

\section{Conclusions}

Taken together, our data revealed that KIAA0101 knockdown hindered cell proliferation, and induced cell cycle arrest and cell death in CLL cells, suggesting the vital roles of KIAA0101 in CLL progression. Also, our study disclosed the main functions and regulatory pathways of KIAA0101, and identified 785 potential targets of KIAA0101 in CLL, which has potential value in elucidating the functions and regulatory mechanisms of KIAA0101 in CLL. In addition, microarray analysis and western blot assays demonstrated that KIAA0101 knockdown led to the notable reduction of FOXO1, MYD 88, and TLR4 levels in CLL cells. KIAA0101-mediated regulation of cell proliferation may be realized through TLR4/MYD88 signaling pathway and FOXO1 expression might be 
independent on cell proliferation in KIAA0101-depleted CLL cells. it is imperative to further investigate the of underlying mechanism KIAA0101 on CLL tumorigenesis and progression. Also, other pivotal targets of KIAA0101 need to be further discovered.

\section{Acknowledgments}

We thank Dr. Wu Kongming for the helpful suggestion. Funding: This work was funded by the Natural Science Foundation of Henan Province China (182300410347), and the National Natural Science Foundation of China (81470336).

\section{Footnote}

Reporting Checklist: The authors have completed the MDAR reporting checklist. Available at http://dx.doi.org/10.21037/ atm-21-626

Data Sharing Statement: Available at http://dx.doi. org/10.21037/atm-21-626

Conflicts of Interest: All authors have completed the ICMJE uniform disclosure form (available at http://dx.doi. org/10.21037/atm-21-626). The authors have no conflicts of interest to declare.

Ethical Statement: The authors are accountable for all aspects of the work in ensuring that questions related to the accuracy or integrity of any part of the work are appropriately investigated and resolved. All procedures performed in this study involving human participants were in accordance with the Declaration of Helsinki (as revised in 2013). The study was approved by the Ethics Committee of the Henan Cancer Hospital (No. 2016098). Written informed consent was obtained from all participants. A copy of the written consent is provided to the editors of this journal for review.

Open Access Statement: This is an Open Access article distributed in accordance with the Creative Commons Attribution-NonCommercial-NoDerivs 4.0 International License (CC BY-NC-ND 4.0), which permits the noncommercial replication and distribution of the article with the strict proviso that no changes or edits are made and the original work is properly cited (including links to both the formal publication through the relevant DOI and the license).
See: https://creativecommons.org/licenses/by-nc-nd/4.0/.

\section{References}

1. Lapierre M, Castet-Nicolas A, Gitenay D, et al. Expression and role of RIP140/NRIP1 in chronic lymphocytic leukemia. J Hematol Oncol 2015;8:20.

2. Cang S, Iragavarapu C, Savooji J, et al. ABT-199 (venetoclax) and BCL-2 inhibitors in clinical development. J Hematol Oncol 2015;8:129.

3. Rai KR. Therapeutic potential of new B cell-targeted agents in the treatment of elderly and unfit patients with chronic lymphocytic leukemia. J Hematol Oncol 2015;8:85.

4. Deeks ED. Ibrutinib: A Review in Chronic Lymphocytic Leukaemia. Drugs 2017;77:225-36.

5. Kwok M, Davies N, Agathanggelou A, et al. ATR inhibition induces synthetic lethality and overcomes chemoresistance in TP53-or ATM-defective chronic lymphocytic leukemia cells. Blood 2016;127:582-95.

6. Wu J, Liu C, Tsui ST, et al. Second-generation inhibitors of Bruton tyrosine kinase. J Hematol Oncol 2016;9:80.

7. Sharma S, Galanina N, Guo A, et al. Identification of a structurally novel BTK mutation that drives ibrutinib resistance in CLL. Oncotarget 2016;7:68833-41.

8. Banerjee R, Russo N, Liu M, et al. TRIP13 promotes error-prone nonhomologous end joining and induces chemoresistance in head and neck cancer. Nat Commun 2014;5:4527.

9. Kang JU, Koo SH, Kwon KC, et al. Gain at chromosomal region $5 \mathrm{p} 15.33$, containing TERT, is the most frequent genetic event in early stages of non-small cell lung cancer. Cancer Genet Cytogenet 2008;182:1-11.

10. Zhou K, Zhang W, Zhang Q, et al. Loss of thyroid hormone receptor interactor 13 inhibits cell proliferation and survival in human chronic lymphocytic leukemia. Oncotarget 2017;8:25469-81.

11. Larkin SET, Holmes S, Cree IA, et al. Identification of markers of prostate cancer progression using candidate gene expression. Br J Cancer 2012;106:157-65.

12. De Biasio A, de Opakua AI, Mortuza GB, et al. Structure of p15(PAF)-PCNA complex and implications for clamp sliding during DNA replication and repair. Nat Commun 2015;6:6439.

13. Zhang T, Guo J, Gu J, et al. KIAA0101 is a novel transcriptional target of FoxM1 and is involved in the regulation of hepatocellular carcinoma microvascular invasion by regulating epithelial-mesenchymal transition. J 
Cancer 2019;10:3501-16.

14. Jain N, Roy J, Das B, et al. miR-197-5p inhibits sarcomagenesis and induces cellular senescence via repression of KIAA0101. Mol Carcinog. 2019;58:1376-88.

15. Wang Z, Dang C, Yan R, et al. Screening of cell cyclerelated genes regulated by KIAA0101 in gastric cancer. Nan Fang Yi Ke Da Xue Xue Bao 2018;38:1151-8.

16. Chen H, Xia B, Liu T, et al. KIAA0101, a target gene of miR-429, enhances migration and chemoresistance of epithelial ovarian cancer cells. Cancer Cell Int 2016;16:74.

17. Melo JV, Foroni L, Brito-Babapulle V, et al. The establishment of cell lines from chronic B cell leukemia: evidence of leukaemic origin by karyotypic abnormalities and Ig gene rearrangement. Clin Exp Immunol 1988;73:23-8.

18. Griggio V, Vitale C, Todaro M, et al. HIF-1 $\alpha$ is overexpressed in leukemic cells from TP53-disrupted patients and is a promising therapeutic target in chronic lymphocytic leukemia. Haematologica 2020;105:1042-54.

19. Lloret A, Fuchsberger T, Giraldo E, et al. Reductive Stress: A New Concept in Alzheimer's Disease. Curr Alzheimer Res 2016;13:206-11.

20. Lv W, Su B, Li Y, et al. KIAA0101 inhibition suppresses cell proliferation and cell cycle progression by promoting the interaction between $\mathrm{p} 53$ and Sp1 in breast cancer. Biochem Biophys Res Commun 2018;503:600-6.

21. Lupi LA, Cucielo MS, Silveira HS, et al. The role of Tolllike receptor 4 signaling pathway in ovarian, cervical, and endometrial cancers. Life Sci 2020;247:117435.

22. Giménez N, Schulz R, Higashi M, et al. Targeting IRAK4 disrupts inflammatory pathways and delays tumor

Cite this article as: Zhang Q, Yuan J, Liu Y, Liu X, Lv T, Zhou K, Song Y. KIAA0101 knockdown inhibits cell proliferation and induces cell cycle arrest and cell apoptosis in chronic lymphocytic leukemia cells. Ann Transl Med 2021;9(6):487. doi: 10.21037/atm-21-626 development in chronic lymphocytic leukemia. Leukemia 2020;34:100-14.

23. Zhu G, Cheng Z, Huang Y, et al. MyD88 mediates colorectal cancer cell proliferation, migration and invasion via NF- $\mathrm{NB} / \mathrm{AP}-1$ signaling pathway. Int J Mol Med 2020;45:131-40.

24. Wu K, Zhang H, Fu Y, et al. TLR4/MyD88 signaling determines the metastatic potential of breast cancer cells. Mol Med Rep 2018;18:3411-20.

25. Farhan M, Wang H, Gaur U, et al. FOXO Signaling Pathways as Therapeutic Targets in Cancer. Int J Biol Sci 2017;13:815-27.

26. Liu DZ, Chang B, Li XD, et al. MicroRNA-9 promotes the proliferation, migration, and invasion of breast cancer cells via down-regulating FOXO1. Clin Transl Oncol 2017;19:1133-40.

27. Gao Z, Liu R, Ye N, et al. FOXO1 Inhibits Tumor Cell Migration via Regulating Cell Surface Morphology in Non-Small Cell Lung Cancer Cells. Cell Physiol Biochem 2018;48:138-48.

28. Choi Y, Park J, Ko YS, et al. FOXO1 reduces tumorsphere formation capacity and has crosstalk with LGR5 signaling in gastric cancer cells. Biochem Biophys Res Commun 2017;493:1349-55.

29. Zhang B, Gui LS, Zhao XL, et al. FOXO1 is a tumor suppressor in cervical cancer. Genet Mol Res 2015;14:6605-16.

30. Cosimo E, Tarafdar A, Moles MW, et al. Akt/mTORC2 inhibition activates FOXO1 function in CLL cells reducing B-cell receptor-mediated survival. Clin Cancer Res 2019;25:1574-87. 